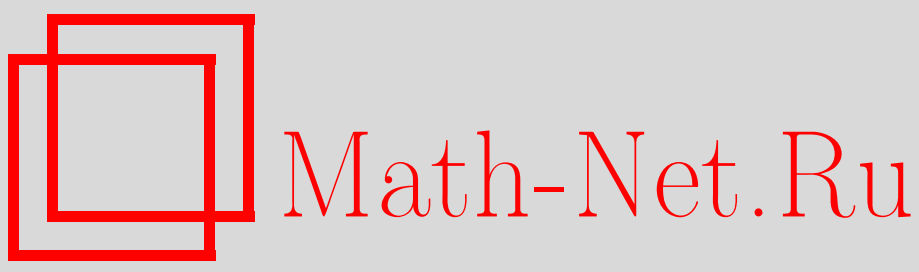

В. А. Смирнов, С. В. Кузнецова, И. В. Майорова, Описание когомологий банаховых и полинормированных алгебр на языке $A_{\infty}$-структур, Изв. РАН. Сер. матем., 1998, том 62, выпуск 4, 155-172

DOI: https://doi.org/10.4213/im197

Использование Общероссийского математического портала Math-Net.Ru подразумевает, что вы прочитали и согласны с пользовательским соглашением

http://www.mathnet.ru/rus/agreement

Параметры загрузки:

IP: 52.205 .19 .152

26 апреля 2023 г., 13:14:40 
УДК 513.83

\author{
В. А. Смирнов, С. В. Кузнецова, И. В. Майорова
}

\title{
Описание когомологий банаховых и полинормированных алгебр на языке $A_{\infty}$-структур
}

\begin{abstract}
Рассматривается вопрос о том, как методы описания когомологий алгебр, основанные на использовании $A_{\infty}$-структур Сташеффа, могут быть применены для описания когомологий банаховых и полинормированных алгебр.

Библиографиия: 4 наименования.
\end{abstract}

Вопрос о вычислении или описании когомологий алгебр является чрезвычайно важным для многих разделов математики. Частным случаем когомологий алгебр являются когомологии дискретных групп, которые играют важную роль в теории групп. Когомологии классифищирующих пространств также являются частным случаем когомологий алгебр и их вычисление необходимо для решения задач дифференщиальной геометрии и топологии, в частности теории кобордизмов гладких многообразий. Когомологии алгебр играют существенную роль в алгебраической топологии и теории гомотопий, поскольку основные спектральные последовательности для вычисления гомотопических групп топологических пространств в качестве начального члена $E^{2}$ имеют когомологии тех или иных алгебр. В частности, член $E^{2}$ спектральной последовательности Адамса стабильных гомотопических групп сфер изоморфен когомологиям алгебры Стинрода.

В то же время проблема вычисления когомологий алгебр является чрезвычайно трудной. Среди обших подходов к ее решению отметим результаты Гугенхейма и Мэя [1], которые показали, что если когомологии алгебры рассматривать не просто как алгебру, а добавить так называемые произведения Масси, то в хороших случаях одномерные когомологии будут содержать все образующие в том смысле, что все остальные элементы в когомологиях могут быть получены из одномерных элементов применением к ним произведений Масси. Одномерные когомологии легко вычисляются, что позволяет практически во всех случаях найти образующие для когомологий алгебр, а остальные элементы получать как произведения Масси от образующих.

Вопрос, который оставался открытым для полного описания когомологий алгебр, заключался в нахождении соотношений между произведениями Масси. Основная трудность состояла в том, что произведения Масси являются частичными и многозначными операциями, и поэтому неясно, как описывать соотношения между ними. Избежать этого помогает язык $A_{\infty}$-структур, введенный Сташеффым [2] и использованный в работе [3] для описания когомологий алгебр, нахождения образуюших и соотношений. Здесь мы рассмотрим вопрос о том, как методы описания

Работа выполнена при финансовой поддержке Российского фонда фундаментальных исследований (грант № 96-01-01049).

(C) В.А. Смирнов, С.В. КУЗНЕцовА, И. В. МАЙоровА 
когомологий алгебр, основанные на использовании $A_{\infty}$-структур Сташеффа, могут быть применены для описания когомологий банаховых и полинормированных алгебр.

Для простоты изложения мы будем рассматривать случай когомологий алгебр с тривиальными коэффициентами в поле комплексных чисел. Вначале напомним необходимые сведения из гомологической алгебры в категории банаховых пространств [4]. Для определенности мы будем рассматривать банаховы пространства, хотя все конструкции могут быть распространены на полные полинормированные алгебры - алгебры Аренса-Майкла.

\section{§1. Комплексы в категории банаховых пространств}

Банаховым комплексом будем называть семейство $X=\left\{X_{n}\right\}_{n \in \mathbb{Z}}$ банаховых пространств $X_{n}$ и непрерывных операторов $d=\left\{d_{n}\right\}, d_{n}: X_{n} \rightarrow X_{n-1}$, называемых дифференииалами и удовлетворяющих соотношениям $d_{n-1} \circ d_{n}=0$. Как правило, мы будем рассматривать банаховы комплексы $X=\left\{X_{n}\right\}$, для которых $X_{n}=0$ при $n<0$ или $X_{n}=0$ при $n>0$.

Банахов комплекс $X$ будем назьвать допусти.мым, если существует семейство непрерывных операторов $s=\left\{s_{n}\right\}, s_{n}: X_{n-1} \rightarrow X_{n}$, удовлетворяющих соотношениям $d_{n} \circ s_{n} \circ d_{n}=d_{n}$.

Пространства $Z_{n}(X)=\operatorname{Ker}\left\{d_{n}: X_{n} \rightarrow X_{n-1}\right\}$ называются пространствами $n$-мерных циклов. Пространства $B_{n}(X)=\operatorname{Im}\left\{d_{n+1}: X_{n+1} \rightarrow X_{n}\right\}$ называются пространствами $n$-мерных грании. Ясно, что $B_{n}(X) \subset Z_{n}(X)$. Факторпространства $H_{n}(X)=Z_{n}(X) / B_{n}(X)$ называется $n$-мернымми гомологиями банахова комплекса $X$. Семейство $H_{*}(X)=\left\{H_{n}(X)\right\}$ называется гомологиями банахова комплекса $X$. В случае если $X$ - допустимьй банахов комплекс, то отображение $d_{n+1} \circ s_{n}: Z_{n}(X) \rightarrow B_{n}(X)$ будет обратным к вложению $i_{n}: B_{n}(X) \rightarrow Z_{n}(X)$. Следовательно, гомологии $H_{*}(X)$ в этом случае будут состоять из банаховых пространств $H_{n}(X)$. Короткие точные последовательности

$$
0 \longrightarrow B_{n}(X) \stackrel{i_{n}}{\longrightarrow} Z_{n}(X) \stackrel{p_{n}}{\longrightarrow} H_{n}(X) \longrightarrow 0
$$

расщепляются, т.е. существуют непрерывные операторы $q_{n}: H_{n}(X) \rightarrow Z_{n}(X)$, для которых выполняются соотношения

$$
p_{n} \circ q_{n}=\mathrm{Id}: H_{n}(X) \rightarrow H_{n}(X), \quad \mathrm{Id}-q_{n} \circ p_{n}=d_{n+1} \circ s_{n}: Z_{n}(X) \rightarrow B_{n}(X)
$$

Для банахова комплекса $X$ обозначим через $\bar{X}$ двойственный комплекс, $\bar{X}=$ $\left\{\bar{X}_{n}\right\}$, для которого $\bar{X}_{n}$ - банахово пространство, сопряженное к $X_{-n}$. Дифференциалы $d_{n}: X_{n} \rightarrow X_{n-1}$ индуцируют дифференциалы $\bar{d}_{n}: \bar{X}_{-n+1} \rightarrow \bar{X}_{-n}$. Ясно, что если $X$ был допустимым банаховым комплексом, то $\bar{X}$ также будет допустимым банаховым комплексом. Гомологии двойственного комплекса $\bar{X}$ называются когомологиями комплекса $X$ и обозначаются $H^{*}(X)=\left\{H^{n}(X)\right\}, H^{n}(X)=$ $H_{-n}(\bar{X})$.

Отображсением банаховых комплексов $f: X \rightarrow Y$ размерности $m$ будем называть семейство $f=\left\{f_{n}\right\}$ непрерывных операторов $f_{n}: X_{n} \rightarrow Y_{n+m}$. 
Ясно, что такие отображения образуют линейное пространство, обозначаемое $\operatorname{Hom}_{m}(X, Y)$.

Определим линейные операторы $d: \operatorname{Hom}_{m}(X, Y) \rightarrow \operatorname{Hom}_{m-1}(X, Y)$, называемые дифференциалами, полагая

$$
d(f)_{n}=d_{n+m} \circ f_{n}+(-1)^{m} f_{n-1} \circ d_{n}: X_{n} \rightarrow Y_{n+m-1} .
$$

Легко видеть, что $d \circ d=0$, и таким образом, семейство $\operatorname{Hom}(X, Y)=$ $\left\{\operatorname{Hom}_{m}(X, Y)\right\}$ образует комплекс в категории линейных пространств.

Цепным отображением банаховых комплексов будем называть отображение $f: X \rightarrow Y$ размерности нуль, удовлетворяюшее соотношению $d(f)=0$, или, что то же самое, $d_{n} \circ f_{n}=f_{n-1} \circ d_{n}$.

Цепное отображение банаховых комплексов $f: X \rightarrow Y$ индуцирует отображение гомологий

$$
H_{*}(f)=\left\{H_{n}(f)\right\}: H_{*}(Y) \rightarrow H_{*}(X), \quad H_{n}(f): H_{n}(Y) \rightarrow H_{n}(X),
$$

и когомологий

$$
H^{*}(f)=\left\{H^{n}(f)\right\}: H^{*}(X) \rightarrow H^{*}(Y), \quad H^{n}(f): H^{n}(X) \rightarrow H^{n}(Y) .
$$

Два цепных отображения $f, g: X \rightarrow Y$ банаховых комплексов называются гомотопными (обозначается $f \simeq g$ ), если сушествует отображение $h: X \rightarrow Y$ размерности 1 , удовлетворяющее соотношению $d(h)=g-f$, или, что то же самое, $d_{n+1} \circ h_{n}+h_{n-1} \circ d_{n}=g_{n}-f_{n}$. Отображение $h$ при этом называется гомотопией между отображениями $f u g$. Легко видеть, что отношение гомотопности является отношением эквивалентности.

Банаховы комплексы $X$ и $Y$ называются гомотопически эквивалентнылми (обозначается $X \simeq Y$ ), если существуют цепные отображения $f: X \rightarrow Y, g: Y \rightarrow$ $X$ такие, что $g \circ f \simeq \operatorname{Id}_{X}, f \circ g \simeq \operatorname{Id}_{Y}$. Банахов комплекс называется стягиваемылм, если он гомотопически эквивалентен нулевому комплексу.

Легко видеть, что если $f \simeq g: X \rightarrow Y$, то $\bar{f} \simeq \bar{g}: \bar{Y} \rightarrow \bar{X}$, и если $X \simeq Y$, то $\bar{X} \simeq \bar{Y}$. Кроме того, гомотопные отображения индуцируют одинаковые гомоморфизмы гомологий и когомологий, а гомотопически эквивалентные банаховы комплексы имеют изоморфные гомологии и когомологии.

ПРЕДЛОЖЕНИЕ 1. Если X - допустимый банахов комплекс, то его гомологии $H_{*}(X)$, рассматриваемые как банахов комплекс с нулевым дифференциалом, будут гомотопически эквивалентны $X: H_{*}(X) \simeq X$.

Действительно, рассмотрим отображение $\mathrm{Id}-s \circ d: X \rightarrow Z(X)$. Его композицию с проекцией $p: Z(X) \rightarrow H_{*}(X)$ обозначим $\eta: X \rightarrow H_{*}(X)$. Рассмотренное вьше отображение $q: H_{*}(X) \rightarrow Z(X)$ дает отображение $\xi: H_{*}(X) \rightarrow X$. Легко видеть, что $\eta \circ \xi=\mathrm{Id}: H_{*}(X) \rightarrow H_{*}(X)$. Покажем, что отображения $s_{n}: X_{n} \rightarrow X_{n+1}$ дают требуемую гомотопию между отображениями $\operatorname{Id}$ и $\xi \circ \eta$. Имеем

$$
\operatorname{Id}-\xi_{n} \circ \eta_{n}=\operatorname{Id}-q_{n} \circ p_{n}\left(\operatorname{Id}-s_{n} \circ d_{n}\right)=d_{n+1} \circ s_{n}+s_{n} \circ d_{n} .
$$

Из этого предложения, в частности получаем 
СлЕДСТВИЕ 1. Для допустимого банахова комплекса $X$ имеет место изоморфизм $H^{*}(X) \cong \overline{H_{*}(X)}$.

Действительно, из гомотопической эквивалентности $X \simeq H_{*}(X)$ следует гомотопическая эквивалентность $\bar{X} \simeq \overline{H_{*}(X)}$, и поэтому имеем указанньй изоморфизм.

Пусть $X$ - банахов комплекс. Определим банахов комплекс $S X$, называемый надстройкой над $X$, полагая $(S X)_{n+1}=X_{n}$. Элементы в $S X$ будем обозначать $[x]$, где $x \in X$. Если $x \in X$ имеет размерность $n$, то $[x]$ будет иметь размерность $n+1$. Дифференциал на $S X$ определим формулой $d[x]=-[d(x)]$.

Ясно, что если $X$ - допустимый банахов комплекс, то надстройка $S X$ будет допустимым банаховым комплексом, и гомологии надстройки изоморфны надстройке над гомологиями, т.е. $H_{*}(S X) \cong S H_{*}(H)$.

Аналогичным образом для банахова комплекса $X$ можно определить денадстройку $S^{-1} X$, полагая $\left(S^{-1} X\right)_{n-1}=X_{n}$. Элементы в денадстройке, когда это не будет вызывать недоразумений, мы будем обозначать $[x]$, где $x \in X$. Если $x \in X$ имеет размерность $n$, то $[x]$ будет иметь размерность $n-1$. Дифференциал на $S X$ определим формулой $d[x]=-[d(x)]$.

Ясно, что функторы надстройки и денадстройки являются взаимно обратньми.

Тензорным произведением банаховых комплексов $X^{\prime}$ и $X^{\prime \prime}$ называется банахов комплекс $X=X^{\prime} \otimes X^{\prime \prime}$, для которого

$$
\left(X^{\prime} \otimes X^{\prime \prime}\right)_{n}=\sum_{p+q=n} X_{p}^{\prime} \otimes X_{q}^{\prime \prime},
$$

и дифференциал определен формулой

$$
d_{n}\left(x_{p}^{\prime} \otimes x_{q}^{\prime \prime}\right)=d_{p}^{\prime}\left(x_{p}^{\prime}\right) \otimes x_{q}^{\prime \prime}+(-1)^{p} x_{p}^{\prime} \otimes d_{q}^{\prime \prime}\left(x_{q}^{\prime \prime}\right), \quad p+q=n .
$$

Для отображений $f^{\prime}: X^{\prime} \rightarrow Y^{\prime}, f^{\prime \prime}: X^{\prime \prime} \rightarrow Y^{\prime \prime}$ размерности $m^{\prime}$ и $m^{\prime \prime}$ соответственно определим отображение $f=f^{\prime} \otimes f^{\prime \prime}: X^{\prime} \otimes X^{\prime \prime} \rightarrow Y^{\prime} \otimes Y^{\prime \prime}$ размерности $m=m^{\prime}+m^{\prime \prime}$, положив на образуюших $x_{p}^{\prime} \otimes x_{q}^{\prime \prime} \in X_{p}^{\prime} \otimes X_{q}^{\prime \prime}$

$$
\left(f^{\prime} \otimes f^{\prime \prime}\right)\left(x_{p}^{\prime} \otimes x_{q}^{\prime \prime}\right)=(-1)^{m^{\prime \prime}} f_{p}^{\prime}\left(x_{p}^{\prime}\right) \otimes f_{q}^{\prime \prime}\left(x_{q}^{\prime \prime}\right) .
$$

Формулу для дифференциала в тензорном произведении банаховых комплексов можно переписать в виде $d=d^{\prime} \otimes 1+1 \otimes d^{\prime \prime}$.

ПРЕДЛОЖЕНИЕ 2. Тензорное произведение допустимых банаховых комплексов является допустимым банаховым комплексом.

Действительно, пусть $\left(X^{\prime}, d^{\prime}, s^{\prime}\right),\left(X^{\prime \prime}, d^{\prime \prime}, s^{\prime \prime}\right)$ - допустимые банаховы комплексы. Определим $s: X^{\prime} \otimes X^{\prime \prime} \rightarrow X^{\prime} \otimes X^{\prime \prime}$, положив

$$
s=s^{\prime} \otimes 1+1 \otimes s^{\prime \prime}-\left(d^{\prime} \circ s^{\prime}+s^{\prime} \circ d^{\prime}\right) \otimes s^{\prime \prime} .
$$

Непосредственные вычисления показывают, что справедливо требуемое соотношение $d \circ s \circ d=d$. 
Если отображения $f^{\prime}: X^{\prime} \rightarrow Y^{\prime}, f^{\prime \prime}: X^{\prime \prime} \rightarrow Y^{\prime \prime}$ соответственно гомотопны отображениям $g^{\prime}: X^{\prime} \rightarrow Y^{\prime}, g^{\prime \prime}: X^{\prime \prime} \rightarrow Y^{\prime \prime}$, то $f^{\prime} \otimes f^{\prime \prime}: X^{\prime} \otimes X^{\prime \prime} \rightarrow Y^{\prime} \otimes Y^{\prime \prime}$ будет гомотопно отображению $g^{\prime} \otimes g^{\prime \prime}: X^{\prime} \otimes X^{\prime \prime} \rightarrow Y^{\prime} \otimes Y^{\prime \prime}$. Требуемая гомотопия $h: X^{\prime} \otimes X^{\prime \prime} \rightarrow Y^{\prime} \otimes Y^{\prime \prime}$ определяется формулой $h=h^{\prime} \otimes g^{\prime \prime}+f^{\prime} \otimes h^{\prime \prime}$, где $h^{\prime}, h^{\prime \prime}-$ соответствуюшие гомотопии между $f^{\prime}, g^{\prime}$ и $f^{\prime}, f^{\prime \prime}$.

Из этого, в частности, следует, что гомотопически эквивалентные цепные комплексы $X^{\prime} \simeq Y^{\prime}, X^{\prime \prime} \simeq Y^{\prime \prime}$ дают гомотопически эквивалентные тензорные произведения $X^{\prime} \otimes X^{\prime \prime} \simeq Y^{\prime} \otimes Y^{\prime \prime}$.

Из предложения 1 получаем

СлеДСТвИЕ 2. Для допустимых банаховых комплексов $X$ и $Y$ имеет место изоморфизм $H_{*}(X \otimes Y) \cong H_{*}(X) \otimes H_{*}(Y)$.

\section{§ 2. Гомологии и когомологии банаховых алгебр}

Пусть $A$ - банахова алгебра, т.е. банахово пространство вместе с ассоциативным умножением $\pi: A \otimes A \rightarrow A$. Рассмотрим комплекс $B A$, называемый $B$-конструкцией над $A$, для которого

$$
(B A)_{n}= \begin{cases}A^{\otimes n}, & n \geqslant 1 \\ 0, & n \leqslant 0 .\end{cases}
$$

Образуюшие элементы размерности $n$ в $A^{\otimes n}$ обозначаются $\left[x_{1}, \ldots, x_{n}\right], x_{i} \in A$. Дифференциал $d$ определен формулами

$$
d_{n}\left[x_{1}, \ldots, x_{n}\right]=\sum_{i=1}^{n-1}(-1)^{i+1}\left[x_{1}, \ldots, \pi\left(x_{i} \otimes x_{i+1}\right), \ldots, x_{n}\right] .
$$

Банахову алгебру $A$ будем называть допустимой, если допустим комплекс $B A$.

Ясно, что любая конечномерная банахова алгебра допустима. В качестве примера бесконечномерной допустимой банаховой алгебры рассмотрим алгебру $A=l_{1}$ абсолютно сходяшихся рядов $\sum_{n=1}^{\infty} x_{n}$ с умножением

$$
\sum_{n=1}^{\infty} x_{n} \cdot \sum_{n=1}^{\infty} y_{n}=\sum_{n=2}^{\infty}\left(\sum_{k=1}^{n-1} x_{k} \cdot y_{n-k}\right) .
$$

Обозначим через $e_{i}$ ряд, у которого на $i$-м месте стоит единица, а на остальных нули. Определим отображения $s: A^{\otimes n} \rightarrow A^{\otimes n+1}$, полагая

$$
s\left(e_{i_{1}} \otimes \cdots \otimes e_{i_{n}}\right)= \begin{cases}e_{1} \otimes e_{i_{1}-1} \otimes \cdots \otimes e_{i_{n}}, & i_{1}>1, \\ 0, & i_{1}=1 .\end{cases}
$$

На остальных элементах $s$ определяется по линейности и непрерывности.

Непосредственные выгисления показьвают, что имеет место соотношение $d \circ s \circ d=d$, означающее, что $A$ - допустимая алгебра.

Среди свойств допустимых банаховых алгебр выделим следующие свойства.

СвойСтво 1. Тензорное произведение допустимых банаховых алгебр является допустимой банаховой алгеброй.

Действительно, пусть $A^{\prime}, A^{\prime \prime}$ - допустимые алгебры, $s^{\prime}: A^{\prime \otimes n} \rightarrow A^{\prime \otimes n+1}, s^{\prime \prime}$ : $A^{\prime \prime \otimes n} \rightarrow A^{\prime \prime \otimes n+1}-$ соответствуюшие отображения в $B$-конструкциях. Тогда искомое отображение $s: A^{\otimes n} \rightarrow A^{\otimes n+1}$ для алгебры $A=A^{\prime} \otimes A^{\prime \prime}$ определяется как тензорное произведение $s^{\prime}$ и $s^{\prime \prime}$. 
Свойство 2. Прямая сумма допустимых банаховых алгебр является допустимой банаховой алгеброй.

Действительно, пусть $A^{\prime}, A^{\prime \prime}$ - допустимые алгебры, $s^{\prime}: A^{\prime \otimes n} \rightarrow A^{\prime \otimes n+1}, s^{\prime \prime}$ : $A^{\prime \prime \otimes n} \rightarrow A^{\prime \prime \otimes n+1}$ - соответствующие отображения в $B$-конструкциях.

Рассмотрим банахову алгебру $A=A^{\prime} \oplus A^{\prime \prime}$. Образующие элементы в $A^{\otimes n}$ представляют собой наборы $a_{1} \otimes \cdots \otimes a_{n}$, где $a_{i}$ принадлежит $A^{\prime}$ или $A^{\prime \prime}$. Разобьем этот набор на блоки $b_{i}$, состояшие из элементов одной и той же алгебры, и перепишем его в виде $b_{1} \otimes \cdots \otimes b_{m}$, где блоки $b_{1}, \ldots, b_{m}$ являются тензорньми произведениями элементов из $A^{\prime}$ или $A^{\prime \prime}$, причем соседние блоки содержат элементы из разных алгебр. Таким образом, $b_{i} \in B A^{\prime}$ или $b_{i} \in B A^{\prime \prime}$.

Определим отображение $s: A^{\otimes n} \rightarrow A^{\otimes n+1}$, полагая

$$
\begin{aligned}
& s\left(b_{1} \otimes \cdots \otimes b_{m}\right)=s\left(b_{1}\right) \otimes b_{2} \otimes \cdots \otimes b_{m} \\
& \quad+(-1)^{n_{1}}\left(b_{1}-d \circ s\left(b_{1}\right)\right) \otimes s\left(b_{2}\right) \otimes \cdots \otimes b_{m}+\ldots \\
& \quad \cdots+(-1)^{n_{1}+\cdots+n_{m-1}}\left(b_{1}-d \circ s\left(b_{1}\right)\right) \otimes \cdots \otimes\left(b_{m-1}-d \circ s\left(b_{m-1}\right)\right) \otimes s\left(b_{m}\right),
\end{aligned}
$$

где $n_{i}$ - размерности $b_{i}$.

Непосредственные вычисления показывают, что справедливы требуемые соотношения.

Гомологии (когомологии) $B$-конструкции $B A$ над алгеброй $A$ называются гомологиями (когомологиями) алгебры $A$ и обозначаются $H_{*}(A)\left(H^{*}(A)\right)$. Таким образом, по определению $H_{*}(A)=H_{*}(B A)\left(H^{*}(A)=H^{*}(B A)\right)$.

Отметим, что гомологии и когомологии уместно рассматривать только для алгебр без единицы. Действительно, если $A$ - алгебра с единицей $e$, то $B$-конструкция $B A$ является стягиваемым комплексом, стягиваюшая гомотопия $h: B A \rightarrow B A$ в котором определена формулой $h\left[x_{1}, \ldots, x_{n}\right]=\left[e, x_{1}, \ldots, x_{n}\right]$. Следовательно, гомологии и когомологии алгебры $A$ тривиальны. Поэтому в случае, если $A$ - алгебра с единицей, рассматривают алгебру $A / \mathbb{C}$, являюшуюся факторизацией алгебры $A$ по единице. Гомологиями (когомологиями) алгебры $A$ считают гомологии (когомологии) алгебры $A / \mathbb{C}$.

Другим вариантом алгебр являются аугментированные алгебры $A$, т.е. такие, для которых задано отображение алгебр $\varepsilon: A \rightarrow \mathbb{C}$, называемое аугментацией. $\mathrm{B}$ этом случае гомологиями (когомологиями) алгебры $A$ считаются гомологии (когомологии) идеала аугментации $I=\operatorname{Ker} \varepsilon$.

Вопрос о гомологической тривиальности (аменабельности) банаховой алгебры достаточно хорошо разработан. В частности, если алгебра $A$ с единицей обладает диагональю, т.е. таким элементом $d \in A \otimes A$, что $a \cdot d=d \cdot a$ для всех $a \in A$ и $\pi(d)$ - единица в $A$, то гомологии алгебры $A$ будут тривиальны.

Примерами таких алгебр являются алгебры $(n \times n)$-матрищ $\mathscr{M}_{n}$. Если через $e_{k l}$ обозначить матрицу с единищей на $(k \times l)$-м месте и нулями на остальных, то диагональю будет элемент

$$
d_{n}=\frac{1}{n} \sum_{k, l=1}^{n} e_{k l} \otimes e_{l k}
$$


Более слабым условием на алгебру $A$, также приводящим к гомологической тривиальности, является наличие аппроксимативной диагонали, т.е. такой направленности $d_{\nu} \in A \otimes A$, что $\lim _{\nu}\left(a \cdot d_{\nu}-d_{\nu} \cdot a\right)=0$ для всех $a \in A$ и $\pi\left(d_{\nu}\right)-$ аппроксимативная единица в $A$.

Примером такой алгебры является алгебра $\mathscr{K}(H)$ компактных операторов в сепарабельном гильбертовом пространстве $H$.

Другим примером гомологически тривиальной банаховой алгебры является алгебра $L_{1}(G)$ на локально компактной аменабельной группе $G$.

В качестве примеров банаховых и полинормированных алгебр с нетривиальными гомологиями рассмотрим следующие.

1. $A=\mathbb{C}$ с тривиальным умножением. В этом случае $(B A)_{n} \cong \mathbb{C}$ и дифференциал тождественно равен нулю. Поэтому гомологии этой алгебры в каждой размерности $n \geqslant 1$ изоморфны $\mathbb{C}$.

2. $A=l_{1}$ - банахова алгебра абсолютно сходящихся рядов. Известно, что одномерные гомологии этой алгебры изоморфны $\mathbb{C}$, а остальные равны нулю.

3. $A=l_{1}^{(m)}$-подалгебра в $l_{1}$, состояшая из рядов, у которых $m$ первых членов равны нулю. Непосредственно из определения гомологий следует, что одномерные гомологии этой алгебры изоморфны $\mathbb{C}^{\times m+1}$.

4. $A=l_{1}^{m}=l_{1} / l_{1}^{(m)}$. Легко видеть, что одномерные гомологии этой алгебры изоморфны $\mathbb{C}$.

5. $A=\mathscr{O}_{z_{0}}(\mathscr{D})$ - полинормированная алгебра аналитических функций в области $\mathscr{D} \subset \mathbb{C}$, обращающихся в нуль в точке $z_{0} \in \mathscr{D}$. Известно, что одномерные гомологии этой алгебры изоморфны $\mathbb{C}$, а остальные равны нулю.

6. $A=\mathscr{O}_{z_{0}}^{(m)}(\mathscr{D})$ - полинормированная алгебра аналитических функций в области $\mathscr{D} \subset \mathbb{C}$, обращающихся в нуль в точке $z_{0}$ вместе со своими производными до $m$-го порядка включительно. Непосредственно из определения гомологий следует, что одномерные гомологии этой алгебры изоморфны $\mathbb{C}^{\times m+1}$.

\section{§ 3. $B$-конструкции для дифференциальных банаховых алгебр}

Банахов комплекс $X$ будем называть дифференииальной банаховой алгеброй, если задано отображение $\pi: X \otimes X \rightarrow X$, называемое умножением, удовлетворяющее соотношению ассоциативности: $\pi(\pi \otimes 1)=\pi(1 \otimes \pi)$.

Двойственным образом, банахов комплекс $Y$ будем называть дифференииальной банаховой коалгеброй, если задано отображение $\nabla: Y \rightarrow Y \otimes Y$, называемое коумножением, удовлетворяюшее соотношению $(\nabla \otimes 1) \nabla=(1 \otimes \nabla) \nabla$.

Ясно, что если $Y$ - дифференциальная банахова коалгебра, то двойственньй комплекс $\bar{Y}$ будет дифференщиальной банаховой алгеброй, умножение в которой определяется как композиция $\bar{Y} \otimes \bar{Y} \stackrel{i}{\longrightarrow} \overline{Y \otimes Y} \stackrel{\bar{\nabla}}{\longrightarrow} \bar{Y}$.

Градуированной банаховой алгеброй (коалгеброй) будем называть дифференциальную банахову алгебру (коалгебру) с нулевым дифференциалом.

Легко видеть, что если $X$ - допустимая дифференциальная банахова алгебра, то ее гомологии $H_{*}(X)$ будут градуированной банаховой алгеброй, умножение 
$\pi_{*}: H_{*}(X) \otimes H_{*}(X) \rightarrow H_{*}(X)$ в которой задается формулой $\pi_{*}=\eta \circ \pi \circ(\xi \otimes \xi)$, где $\xi: H_{*}(X) \rightarrow X, \eta: X \rightarrow H_{*}(X), \pi: X \otimes X \rightarrow X$.

Двойственным образом, гомологии допустимой дифференциальной банаховой коалгебры являются градуированной банаховой коалгеброй. В частности, гомологии $H_{*}(Y)$ допустимой банаховой коалгебры $Y$ будут градуированной банаховой коалгеброй, а когомологии $H^{*}(Y)$ - градуированной банаховой алгеброй.

Для дифференциальной банаховой алгебры $X$ определим дифференциальную коалгебру $B X$, называемую $B$-конструкиией над $X$. Для этого рассмотрим тензорную алгебру $T X=\sum_{n \geqslant 1} X^{\otimes n}$. Элементы $x_{1} \otimes \cdots \otimes x_{n} \in X^{\otimes n}$ обозначают $\left[x_{1}, \ldots, x_{n}\right]$ и их размерностью считают число, равное сумме размерностей элементов $x_{i}$ плюс $n$. Дифференциал определен формулой

$$
\begin{aligned}
d\left[x_{1}, \ldots, x_{n}\right]= & -\sum_{i=1}^{n}(-1)^{\varepsilon_{i-1}}\left[x_{1}, \ldots, d\left(x_{i}\right), \ldots, x_{n}\right] \\
& +\sum_{i=1}^{n-1}(-1)^{\varepsilon_{i}}\left[x_{1}, \ldots, \pi\left(x_{i} \otimes x_{i+1}\right), \ldots, x_{n}\right],
\end{aligned}
$$

где $\varepsilon_{i}=\operatorname{dim}\left(x_{1}\right)+\cdots+\operatorname{dim}\left(x_{i}\right)+i$.

Тензорная алгебра $T X$ с такой градуировкой и дифференциалом называется $B$-конструкцией над $X$ и обозначается $B X$. Помимо структуры цепного комплекса на $B X$ имеется коумножение $\nabla: B X \rightarrow B X \otimes B X$, преврашаюшее ее в дифференциальную коалгебру,

$$
\nabla\left[x_{1}, \ldots, x_{n}\right]=\sum_{i=1}^{n-1}\left[x_{1}, \ldots, x_{i}\right] \otimes\left[x_{i+1}, \ldots, x_{n}\right] .
$$

Двойственным образом, для дифференциальной банаховой коалгебры $Y$ определена дифференциальная алгебра $F Y$, называемая ко-B-конструкцией над $Y$. Она является тензорной алгеброй $T Y$, элементы $y_{1} \otimes \cdots \otimes y_{n}$ в которой обозначаются $\left[y_{1}, \ldots, y_{n}\right]$ и имеют размерность, равную сумме размерностей элементов $y_{i}$ минус $n$. Дифференциал определен формулой

$$
\begin{aligned}
d\left[y_{1}, \ldots, y_{n}\right]= & -\sum_{i=1}^{n}(-1)^{\varepsilon_{i-1}}\left[y_{1}, \ldots, d\left(y_{i}\right), \ldots, y_{n}\right] \\
& +\sum_{i=1}^{n-1}(-1)^{\varepsilon_{i}}\left[y_{1}, \ldots, x_{i}^{\prime}, x_{i}^{\prime \prime}, \ldots, x_{n}\right],
\end{aligned}
$$

где $\nabla\left(y_{i}\right)=\sum y_{i}^{\prime} \otimes y_{i}^{\prime \prime}$.

Умножение $\pi: F Y \otimes F Y \rightarrow F Y$ определено формулой

$$
\pi\left(\left[y_{1}, \ldots, y_{n}\right] \otimes\left[y_{n+1}, \ldots, y_{n+m}\right]\right)=\left[y_{1}, \ldots, y_{n+m}\right] .
$$

ПРЕДЛОЖЕНИЕ 3. Для произвольной дифференциальной банаховой алгебpы $X$ имеет место гомотопическая әквивалентность $F B X \simeq X$.

Определим отображение $\xi: X \rightarrow F B X$, положив $\xi(x)=[[x]]$, и отображение алгебр $\eta: F B X \rightarrow X$, положив на образуюших $\eta\left[\left[x_{1}, \ldots, x_{n}\right]\right]=x_{1}$, если $n=1$, и 
нуль в остальных случаях. Ясно, что имеет место формула $\eta \circ \xi=\mathrm{Id}: X \rightarrow X$. Покажем, что $\xi \circ \eta: F B X \rightarrow F B X$ гомотопно тождественному отображению. Определим гомотопию $h: F B X \rightarrow F B X$, положив

$h\left(\left[\left[x_{1}\right],\left[x_{2}, \ldots, x_{n}\right], \ldots,\left[x_{m+1}, \ldots, x_{k}\right]\right]\right)=\left[\left[x_{1}, x_{2}, \ldots, x_{n}\right], \ldots,\left[x_{m+1}, \ldots, x_{k}\right]\right]$,

и нуль в остальных случаях. Непосредственные вычисления показывают, что $h$ является искомой гомотопией.

Для дифференциальной полинормированной алгебры $X$ рассмотрим убывающую фильтрацию

$$
X=X^{1} \supset X^{2}=X \cdot X \supset \cdots \supset X^{n} \supset \cdots
$$

и обозначим $\widehat{X}=\lim X / X^{n}$.

$\widehat{X}$ является полинормированной алгеброй, и имеет место естественное отображение алгебр $X \rightarrow \widehat{X}$. Алгебру $X$ будем называть совершенной, если это отображение - изоморфизм.

ПРЕДЛОЖЕНИЕ 4. Для совериенной алгебры $X$ имеет место гомотопическая эквивалентность алгебр $\widehat{F} B X \simeq X$.

Действительно, отображение алгебр $\eta: F B X \rightarrow X$ индуцирует отображение $\hat{\eta}: \widehat{F} B X \rightarrow X$. Кроме того, имеет место вложение $\hat{\xi}: X \rightarrow \widehat{F} B X, \hat{\eta} \circ \hat{\xi}=\mathrm{Id}$. Рассмотренная ранее гомотопия $h: F B X \rightarrow F B X$ сохраняет фильтрацию и, следовательно, индуцирует гомотопию $\hat{h}: \widehat{F} B X \rightarrow \widehat{F} B X, d(\hat{h})=\hat{\xi} \circ \hat{\eta}-\mathrm{Id}$.

Рассмотрим теперь двойственную ситуацию. Для полинормированной коалгебры $Y$ определим фильтрацию $Y^{1} \subset Y^{2} \subset \cdots \subset Y^{n} \subset \cdots \subset Y$, полагая

$$
Y^{1}=P Y=\{y \in Y: \nabla(y)=0\}, \quad Y^{2}=\left\{y \in Y: \nabla(y) Y^{1} \otimes Y^{1}\right\},
$$

и так далее.

Обозначим через $\breve{Y}$ прямой предел $Y^{n}, \breve{Y}=\underline{\lim } Y^{n}$. Ясно, что $\breve{Y}$ будет подкоалгеброй коалгебры $Y$. Коалгебру $Y$ будем называть совершенной, если $\breve{Y}=Y$.

Имеет место следующее предложение, двойственное к предложению 4.

ПРЕДЛОЖЕНИЕ $4^{\prime}$. Для совершенной коалгебры $Y$ имеет место гомотопическая әквивалентность коалгебр $B F Y \simeq Y$.

\section{§4. Дифференциальные $A_{\infty}$-алгебры и $A_{\infty}$-коалгебры}

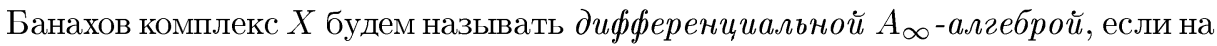
нем заданы операции $\pi_{n}: X^{\otimes n+2} \rightarrow X$ размерности $n \geqslant 0$, удовлетворяющие соотношениям

$$
d\left(\pi_{n+1}\right)=\sum_{i=0}^{n}(-1)^{\varepsilon} \pi_{i}\left(1 \otimes \cdots \otimes \pi_{n-i} \otimes \cdots \otimes 1\right),
$$


где $\varepsilon=n k+i k+n+k$.

Например, если $n=0$, то для операции $\pi_{0}: X_{*} \otimes X_{*} \rightarrow X_{*}$ эта формула дает соотношение гомотопической ассоциативности

$$
d\left(\pi_{1}\right)=\pi_{0}\left(-\pi_{0} \otimes 1+1 \otimes \pi_{0}\right) .
$$

Если $n=1$, то мы имеем соотношение

$$
d\left(\pi_{2}\right)=-\pi_{0}\left(\pi_{1} \otimes 1+1 \otimes \pi_{1}\right)+\pi_{1}\left(\pi_{0} \otimes 1 \otimes 1-1 \otimes \pi_{0} \otimes 1+1 \otimes 1 \otimes \pi_{0}\right),
$$

которое является в некотором смысле соотношением гомотопической ассоциативности между $\pi_{0}$ и $\pi_{1}$. И так далее.

Градуированной банаховой алгеброй будем назьвать дифференциальную банахову алгебру с нулевым дифференциалом.

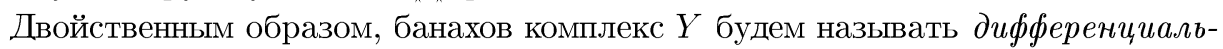
ной $A_{\infty}$-коалгеброй, если на нем заданы операции $\nabla_{n}: Y \rightarrow Y^{\otimes n+2}$ размерности $n \geqslant 0$, удовлетворяющие соотношениям

$$
d\left(\nabla_{n+1}\right)=\sum_{i=0}^{n}(-1)^{\varepsilon}\left(1 \otimes \cdots \otimes \nabla_{n-i} \otimes \cdots \otimes 1\right) \nabla_{i},
$$

где $\varepsilon=n k+i k+n i+i+k+1$.

Для того чтобы избежать трудностей с определением знаков, мы переформулируем определения дифференциальных $A_{\infty}$-алгебры и $A_{\infty}$-коалгебры. А именно, пусть $X$ - банахов комплекс. Рассмотрим надстройку $S X$ и тензорную алгебру

$$
T S X=\sum_{n \geqslant 1}(S X)^{\otimes n}
$$

Элементы в $T S X$ будем обозначать $\left[x_{1}, \ldots, x_{n}\right], x_{i} \in A$. Они имеют размерность, равную сумме размерностей $x_{i}$ плюс $n . T S X$ является коалгеброй, коумножение $\nabla: T S X \rightarrow T S X \otimes T S X$ в которой определяется формулой

$$
\nabla\left[x_{1}, \ldots, x_{n}\right]=\sum_{i=1}^{n-1}\left[x_{1}, \ldots, x_{i}\right] \otimes\left[x_{i+1}, \ldots, x_{n}\right] .
$$

Градуированной банаховой коалгебройбудем назьвать дифференциальную банахову коалгебру с нулевым дифференциалом.

Легко видеть, что задание дифференциала в $T S X$, преврашающего ее в дифференциальную коалгебру, равносильно заданию дифференциала в $S X$ и операций $\pi(i):(S X)^{\otimes i} \rightarrow S X$, понижаюших размерность на единицу и удовлетворяющих соотношениям

$$
d(\pi(n))+\sum_{i=2}^{n-1} \pi(i)(1 \otimes \cdots \otimes \pi(n-i+1) \otimes \cdots \otimes 1)=0 .
$$


Рассмотренное семейство операций обозначим через $\pi=\{\pi(i)\}$ и определим семейство операций $\pi \cup \pi$, положив

$$
\pi \cup \pi(n)=\sum_{i=2}^{n-1} \pi(i)(1 \otimes \cdots \otimes \pi(n-i+1) \otimes \cdots \otimes 1) .
$$

Тогда соотношение для структуры $A_{\infty}$-алгебры можно переписать в виде $d(\pi)+$ $\pi \cup \pi=0$.

Покажем, что задание семейства операций $\pi=\{\pi(i)\}$ равносильно заданию на $X$ структуры дифференциальной $A_{\infty}$-алгебры. Действительно, рассмотрим взаимно обратные отображения $\xi: X \rightarrow S X$ и $\eta: S X \rightarrow X$. Если на $X$ задана структура дифференциальной $A_{\infty}$-алгебры, состояшая из операций $\pi_{i}$ : $X^{\otimes i+2} \rightarrow X$, то в качестве операций $\pi(i+2):(S X)^{\otimes i+2} \rightarrow S X$ возьмем такие операции, чтобы выполнялись равенства $\eta \circ \pi(i+2)=\pi_{i} \circ \eta^{\otimes i+2}$. Имеем

$$
\begin{aligned}
& \left\{d\left(\pi_{n+1}\right)+\sum_{i=0}^{n}(-1)^{n k+i k+n+k} \pi_{i}\left(1 \otimes \cdots \otimes \pi_{n-i} \otimes \cdots \otimes 1\right)\right\} \eta^{\otimes n+3} \\
& \quad=d\left(\pi_{n+1} \eta^{\otimes n+3}\right)+\sum_{i=0}^{n}(-1)^{i-k} \pi_{i}(\eta \otimes \cdots \otimes \eta \pi(n-i+2) \otimes \cdots \otimes \eta) \\
& \quad=\eta\left\{\pi(n+3)+\sum_{i=0}^{n} \pi(i+2)(1 \otimes \cdots \otimes \pi(n-i+2) \otimes \cdots \otimes 1)\right\} .
\end{aligned}
$$

Отсюда следует, что первое выражение равно нулю тогда и только тогда, когда равно нулю третье выражение.

Дифференциальная коалгебра $T S X$, построенная для дифференциальной $A_{\infty}$-алгебры $X$ называется $B$-конструкцией над $X$ и обозначается $B X$. Легко видеть, что в случае, если высшие операции $\pi_{i}$ при $i \geqslant 1$ тривиальны, т.е. $X$ является обычной дифференциальной алгеброй, то $B$-конструкция $B X$ совпадает с обычной $B$-конструкцией $B X$ над алгеброй $X$.

Двойственным образом, для банахова комплекса $Y$ рассмотрим денадстройку $S^{-1} Y$ и тензорную алгебру $\widehat{T} S^{-1} Y$. Тогда задание на $Y$ структуры дифференциальной $A_{\infty}$-коалгебры равносильно заданию в $\widehat{T} S^{-1} Y$ дифференциала, превращающего ее в дифференциальную алгебру, которая называется $к о-B$-конструкцией над $Y$ и обозначается $\widehat{F} Y$. В случае если $Y$ является обычной дифференциальной банаховой коалгеброй, т.е. высшие операции $\nabla_{i}$ при $i \geqslant 1$ тривиальны, то эта ко- $B$-конструкция совпадает с обычной ко- $B$-конструкцией $\widehat{F} Y$ над $Y$.

Пусть $X^{\prime}, X^{\prime \prime}$ - дифференциальные $A_{\infty}$-алгебры. Отображением $A_{\infty}$-алгебр $f: X^{\prime} \rightarrow X^{\prime \prime}$ называется семейство отображений $f_{i}: X^{\prime \otimes i+1} \rightarrow X^{\prime \prime}$ размерности $i$, для которых соответствующие отображения $f(i+1):\left(S X^{\prime}\right)^{\otimes i+1} \rightarrow S X^{\prime \prime}$ удовлетворяют соотношениям

$$
\begin{aligned}
d(f(n))= & \sum_{i=1}^{n-1} f(i)\left(1 \otimes \cdots \otimes \pi^{\prime}(n-i+1) \otimes \cdots \otimes 1\right) \\
& -\sum_{n_{1}+\cdots+n_{i}=n} \pi^{\prime \prime}(i)\left(f\left(n_{1}\right) \otimes \cdots \otimes f\left(n_{i}\right)\right) .
\end{aligned}
$$


Легко видеть, что задание таких операций равносильно заданию отображения дифференциальных банаховых коалгебр $B(f): B X^{\prime} \rightarrow B X^{\prime \prime}$.

Используя произведения семейств $f \cup \pi^{\prime}$ и $\pi^{\prime \prime} \cup f$, соотношения для отображения $A_{\infty}$-алгебр можно переписать в виде $d(f)=f \cup \pi^{\prime}-\pi^{\prime \prime} \cup f$.

Отображения $f, g: X^{\prime} \rightarrow X^{\prime \prime}$ дифференциальных $A_{\infty}$-алгебр называются гомотопными (обозначается $f \simeq g$ ), если сушествует семейство $h=\{h(i)\}$, для которого выполняется соотношение $d(h)=g-f-\pi^{\prime \prime} \cup h-h \cup \pi^{\prime}$. Семейство $h$ называется гомотопией между $f u g$. Легко проверяется, что если $f$ и $g$ гомотопны, то соответствующие отображения $B$-конструкций $B f, B g: B X^{\prime} \rightarrow B X^{\prime \prime}$ также будут гомотопны. Дифференциальные $A_{\infty}$-алгебры $X^{\prime}, X^{\prime \prime}$ называются гомотопически эквивалентными и обозначается $X^{\prime} \simeq X^{\prime \prime}$, если сушествуют отображения $f: X^{\prime} \rightarrow X^{\prime \prime}, g: X^{\prime \prime} \rightarrow X^{\prime}$, для которых $g \circ f \simeq \operatorname{Id}_{X^{\prime}}, f \circ g \simeq \operatorname{Id}_{X^{\prime \prime}}$.

Двойственным образом определяются понятия отображения дифференциальных $A_{\infty}$-коалгебр, гомотопности и гомотопической эквивалентности дифференциальных $A_{\infty}$-коалгебр.

Теорема 1. Если $X$ - допустимая дифференииальная банахова алгебра, то на ее гомологиях $X_{*}=H_{*}(X)$ имеется структура градуированной $A_{\infty}$-алгебры и гомотопическая эквивалентность $A_{\infty}$-алгебр $X \simeq X_{*}$, индуцирующая гомотопическую эквивалентность дифференциальных коалгебр $B X \simeq B X_{*}$.

Действительно, рассмотрим отображения $\xi: X_{*} \rightarrow X, \eta: X \rightarrow X_{*}$ и гомотопию $h: X \rightarrow X$ такие, что

$$
\eta \circ \xi=\mathrm{Id}, \quad d(h)=\xi \circ \eta-\mathrm{Id}, \quad h \circ \xi=0, \quad \eta \circ h=0, \quad h \circ h=0,
$$

и определим структуру $A_{\infty}$-алгебры $\pi=\{\pi(i)\}$, положив

$$
\pi(i)=\eta \pi(h \pi \otimes 1+1 \otimes h \pi) \ldots(h \pi \otimes \cdots \otimes 1+\cdots+1 \otimes \cdots \otimes h \pi)(\xi \otimes \cdots \otimes \xi) .
$$

Непосредственные вычисления показывают, что имеют место требуемые соотношения.

Определим также отображения $\xi_{i}: X_{*}^{\otimes i+1} \rightarrow X, \eta_{i}: X^{\otimes i+1} \rightarrow X_{*}$ и гомотопию $h_{i}: X^{\otimes i+1} \rightarrow X$, положив

$$
\begin{aligned}
& \xi(i)=\pi(h \pi \otimes 1+1 \otimes h \pi) \ldots(h \pi \otimes \cdots \otimes 1+\cdots+1 \otimes \cdots \otimes h \pi)(\xi \otimes \cdots \otimes \xi), \\
& \eta(i)=\eta \pi(h \pi \otimes 1+1 \otimes h \pi) \ldots(h \pi \otimes \xi \eta \otimes \cdots \otimes \xi \eta+\cdots+1 \otimes \cdots \otimes 1 \otimes h \pi), \\
& h(i)=h \pi(h \pi \otimes 1+1 \otimes h \pi) \ldots(h \pi \otimes \xi \eta \otimes \cdots \otimes \xi \eta+\cdots+1 \otimes \cdots \otimes 1 \otimes h \pi) .
\end{aligned}
$$

Непосредственные вычисления показывают, что имеют место требуемые соотношения.

Двойственным образом, имеет место

Теорема 1'. Если $Y$ - допустимая дифференциальная банахова коалгебра, то на ее гомологиях $Y_{*}=H_{*}(Y)$ имеется структура градуированной $A_{\infty}$-коалгебры и гомотопическая әквивалентность $A_{\infty}$-коалгебр $Y \simeq Y_{*}$, индуцирующая гомотопическую әквивалентность дифференциальных алгебр $\widehat{F} Y \simeq \widehat{F} Y_{*}$.

В частности, на гомологиях $H_{*}(A)$ допустимой банаховой алгебры $A$ имеется структура $A_{\infty}$-коалгебры, а на когомологиях $H^{*}(A)$ - структура $A_{\infty}$-алгебры. 


\section{§5. Описание градуированных банаховых $A_{\infty}$-алгебр}

Рассмотрим теперь вопрос о том, что означает описание $A_{\infty}$-алгебр и $A_{\infty}$-коалгебр. Обычные алгебры описываются заданием неразложимых элементов и соотношений между их произведениями. Но что означает неразложимость в случае $A_{\infty}$-алгебр? Неправильным было бы определить разложимые элементы в $A_{\infty}$-алгебре $A$, как те элементы, которые принадлежат образам некоторых произведений $\pi_{n}: A^{\otimes n+2} \rightarrow A$. Для того чтобы дать правильное определение, нужно просто переформулировать определение разложимых элементов для алгебр с произведениями Масси на случай $A_{\infty}$-алгебр. Для упрошения знаков, как и раньше, перейдем к надстройкам.

Пусть $A-A_{\infty}$-алгебра. Последовательность $\left(x^{2}, \ldots, x^{n}\right)$ элементов $x^{i} \in S A^{\otimes i}$ называется последовательностью Масси [3], если выполнены соотношения

$$
\begin{aligned}
& (\pi(2) \otimes \cdots \otimes 1+\cdots+1 \otimes \cdots \otimes \pi(2))\left(x^{n}\right)=0, \\
& (\pi(3) \otimes \cdots \otimes 1+\cdots+1 \otimes \cdots \otimes \pi(3))\left(x^{n}\right) \\
& +(\pi(2) \otimes \cdots \otimes 1+\cdots+1 \otimes \cdots \otimes \pi(2))\left(x^{n-1}\right)=0, \\
& (\pi(n-1) \otimes 1+1 \otimes \pi(n-1))\left(x^{n}\right) \\
& +(\pi(n-2) \otimes 1+1 \otimes \pi(n-2))\left(x^{n-1}\right)+\cdots \\
& \cdots+(\pi(2) \otimes 1+1 \otimes \pi(2))\left(x^{3}\right)=0 .
\end{aligned}
$$

Для таких последовательностей Масси $\left(x^{2}, \ldots, x^{n}\right)$ определяется произведение Масси $\mu\left(x^{2}, \ldots, x^{n}\right)=\pi(2)\left(x^{2}\right)+\cdots+\pi(2)\left(x^{n}\right)$. Элемент $x \in A$ называется разложимым, если он является образом какого-нибудь произведения Масси. Фактор $A$ по разложимым элементам называется модулем неразложимых әлементов и обозначается $J A$.

Переведем это определение на язык $B$-конструкций. Рассмотрим $B$-конструкцию $B A$ над $A_{\infty}$-алгеброй $A$. Вложение $i: A \rightarrow B A$ индуцирует короткую точную последовательность

$$
0 \longrightarrow A \stackrel{i}{\longrightarrow} B A \stackrel{p}{\longrightarrow} B^{1} A=B A / A \longrightarrow 0
$$

и длинную точную последовательность гомологий

$$
\cdots \longrightarrow A \stackrel{i_{*}}{\longrightarrow} H_{*}(B A) \stackrel{p_{*}}{\longrightarrow} H_{*}\left(B^{1} A\right) \stackrel{\mu_{*}}{\longrightarrow} A \longrightarrow \cdots
$$

Из определения последовательности Масси следует, что последовательность $\left(x^{2}, \ldots, x^{n}\right)$ является последовательностью Масси в том и только том случае, когда элемент $x=x^{2}+\cdots+x^{n}$ является циклом в $B^{1}(A)$. Более того, отображение $\mu_{*}$ индуцируется произведением Масси. Таким образом, элемент $x \in A$ разложим тогда и только тогда, когда он принадлежит образу $\mu_{*}$. Следовательно, модуль неразложимых элементов $J A$ изоморфен образу отображения $i_{*}: A \rightarrow H_{*}(B A)$.

Двойственным образом, для градуированной $A_{\infty}$-коалгебры $K$ рассмотрим ко- $B$-конструкцию $\widehat{F} K$. Проекция $p: \widehat{F} K \rightarrow K$ индуцирует короткую точную последовательность

$$
0 \longrightarrow \widehat{F}^{1} K \stackrel{i}{\longrightarrow} \widehat{F} K \stackrel{p}{\longrightarrow} K \longrightarrow 0
$$


и длинную точную последовательность гомологий

$$
\cdots \longrightarrow H_{*}\left(\widehat{F}^{1} K\right) \stackrel{i_{*}}{\longrightarrow} H_{*}(\widehat{F} K) \stackrel{p_{*}}{\longrightarrow} K \stackrel{\nu}{\longrightarrow} H_{*}\left(\widehat{F}^{1} K\right) \longrightarrow \cdots
$$

Элемент $x \in K$ называется примитивным, если он принадлежит ядру гомоморфизма $\nu_{*}: K \rightarrow H_{*}\left(\widehat{F}^{1} K\right)$, или, что то же самое, образу гомоморфизма $p_{*}: H_{*}(\widehat{F} K) \rightarrow K$. Модуль примитивных элементов $A_{\infty}$-коалгебры $K$ обозначим $P K$. Заметим, что в случае обычной коалгебры $K$ с коумножением $\nabla: K \rightarrow$ $K \otimes K$ примитивными элементами являются элементы из ядра отображения $\nabla$.

Перейдем теперь к вопросу об описании соотношений в $A_{\infty}$-алгебре. Пусть $A-$ градуированная $A_{\infty}$-алгебра. Всякое соотношение в $A$ может быть записано в виде

$$
\mu\left(x^{2}, \ldots, x^{n}\right)=\pi(2)\left(x^{2}\right)+\cdots+\pi(n)\left(x^{n}\right)=0,
$$

где $\left(x^{2}, \ldots, x^{n}\right)$ - последовательность Масси и $\mu\left(x^{2}, \ldots, x^{n}\right)$ - произведение Масси. Найти соотношения в $A_{\infty}$-алгебре означает найти такие последовательности Масси, произведения Масси от которых равны нулю. Конечно, некоторые из таких соотношений следуют из структуры $A_{\infty}$-алгебры, и поэтому достаточно находить только такие соотношения, которые не следуют просто из $A_{\infty}$-структуры.

Как мы видели раньше, последовательности Масси $\left(x^{2}, \ldots, x^{n}\right)$ определяют циклы в $B^{1} A$. Покажем, что гомологичные нулю циклы определяют соотношения, которые следуют из $A_{\infty}$-структуры в $A$.

Пусть $y \in S A^{\otimes n+1}, d(y)=x^{2}+\cdots+x^{n}$. Тогда выполняются равенства

$$
\begin{gathered}
x^{2}=(\pi(n) \otimes 1+1 \otimes \pi(n))(y), \\
\cdots \cdots \cdots \cdots \cdots \cdots \cdots \cdots \cdots \cdots \cdots \cdots \cdots \cdots \cdots \cdots \cdots \cdots \cdots+1 \otimes \cdots \otimes 1 \otimes \pi(2))(y) . \\
x^{n}=(\pi(2) \otimes 1 \otimes \cdots \otimes 1+\cdots+1 \otimes \cdots \otimes
\end{gathered}
$$

Последовательность Масси $\left(x^{2}, \ldots, x^{n}\right)$ в этом случае определяет соотношение

$$
\sum \pi(i+2)(1 \otimes \cdots \otimes \pi(n-i) \otimes \cdots \otimes 1)(y)=0,
$$

которое следует непосредственно из $A_{\infty}$-структуры. Таким образом, соотношения, в которых мы нуждаемся, определяются элементами $x$ в гомологиях $H_{*}\left(B^{1} A\right)$, для которых $\mu_{*}(x)=0$, где $\mu_{*}: H_{*}\left(B^{1} A\right) \rightarrow A$.

$\mathrm{C}$ другой стороны, пусть $A_{\infty} A$ обозначает свободную $A_{\infty}$-алгебру, порожденную $A$, и $A_{\infty}^{1} A=A_{\infty} A / A$. Тогда найти соотношения в $A$ означает найти такие элементы $x \in A_{\infty}^{1} A$, для которых $\mu(x)=0$, где $\mu: A_{\infty}^{1} A \rightarrow A$ - отображение, индуцированное структурой $A_{\infty}$-алгебры в $A$. Конечно, некоторые из таких соотношений следуют просто из $A_{\infty}$-структуры в $A$. Для их нахождения рассмотрим отображение $\gamma \times 1-1 \times \mu: A_{\infty} A_{\infty}^{1} A \rightarrow A_{\infty}^{1} A$. Если $x$ принадлежит образу этого отображения, то он порождает соотношение $\mu(x)=0$, которое следует из $A_{\infty}$-структуры в $A$. Обозначим $A_{\infty}^{1} * A$ фактор $A_{\infty}^{1} A$ по образу отображения $\gamma \times 1-1 \times \mu$. Тогда для нахождения соотношений достаточно найти такие элементы $x$ в $A_{\infty}^{1} * A$, которые отображаются в нуль посредством отображения $A_{\infty}^{1} * A \rightarrow A$. 
Для $A_{\infty}$-алгебры $A$ обозначим для краткости гомологии ее $B$-конструкции через $K$, т.е. $K=H_{*}(B A)$. Пусть далее $\bar{A}_{\infty} K$ обозначает свободную $A_{\infty}$-коалгебру, порожденную $K ; \bar{A}_{\infty}^{1} K=\bar{A}_{\infty} K / K$. Структура $A_{\infty}$-коалгебры в $K$ индуцирует отображение $\tau: K \rightarrow \bar{A}_{\infty}^{1} K$. Кроме этого имеется отображение двойственности $\psi: \bar{A}_{\infty}^{1} K \rightarrow A_{\infty}^{1} A$ (см. [3]). Рассмотрим отображение $K \rightarrow A_{\infty}^{1} * A$, являющееся композицией отображений $\tau, \psi$ и проекции $A_{\infty}^{1} A \rightarrow A_{\infty}^{1} * A$. Тогда имеет место коммутативная диаграмма

$$
\begin{gathered}
K \longrightarrow H_{*}\left(B^{1} A\right) \longrightarrow A \\
\downarrow \\
A_{\infty}^{1} * A
\end{gathered}
$$

Из этой диаграммы следует, что для нахождения соотношений в $A_{\infty}$-алгебре $A$ нужно найти образ отображения $K \rightarrow A_{\infty}^{1} * A$. Подводя итог вышесказанному, получаем, что для описания $A_{\infty}$-алгебры $A$ нужно:

$1)$ найти гомологии $H_{*}(B A) B$-конструкции $B A$ над алгеброй $A$;

2 ) вычислить образ гомоморфизма $A \rightarrow H_{*}(B A)$; этот образ будет модулем $J A$ неразложимых элементов $A_{\infty}$-алгебры $A$;

3 в вычислить образ гомоморфизма $H_{*}(B A) \rightarrow A_{\infty}^{1} * A$; этот образ будет давать соотношения в $A_{\infty}$-алгебре $A$.

Двойственным образом, для описания $A_{\infty}$-коалгебры $K$ нужно:

$\left.1^{\prime}\right)$ найти гомологии $H_{*}(\widehat{F} K)$ ко- $B$-конструкции $\widehat{F} K$ над коалгеброй $K$;

$\left.2^{\prime}\right)$ вычислить образ гомоморфизма $H_{*}(\widehat{F} K) \rightarrow K$; этот образ будет модулем примитивных элементов $A_{\infty}$-коалгебры $K$;

$\left.3^{\prime}\right)$ вычислить ядро гомоморфизма $\bar{A}_{\infty}^{1} * K \rightarrow H_{*}(\widehat{F} K)$; это ядро будет давать порождаюшие элементы в искомой $A_{\infty}$-коалгебре.

\section{§6. Нахождение образующих и соотношений в когомологиях банаховых алгебр}

Для описания $A_{\infty}$-алгебр и коалгебр в первую очередь нужно найти неразложимые и примитивные элементы соответственно.

Рассмотрим алгебру $A$ и найдем примитивные элементы гомологий ее $B$-конструкции $H_{*}(B A)$. Имеет место следующая

ТЕОРема 2. Для совершенной алгебры А пространство примитивных әлементов $P H_{*}(B A)$ гомологий $B$-конструкции $B A$ изоморфно пространству неразложимых әлементов алгебры $A: P H_{*}(B A) \cong J A$.

Действительно, по определению примитивных элементов имеем

$$
P H_{*}(B A)=\operatorname{Im}\left\{H_{*}\left(\widehat{F} H_{*}(B A)\right) \rightarrow H_{*}(B A)\right\} .
$$

Используя изоморфизм $H_{*}(\widehat{F} B A) \cong A$, получаем требуемый изоморфизм

$$
P H_{*}(B A) \cong \operatorname{Im}\left\{A \rightarrow H_{*}(B A)\right\} \cong J A .
$$

Рассмотрим теперь коалгебру $K$ и найдем пространство неразложимых элементов $J H_{*}(F K)$ гомологий ее ко- $B$-конструкции $F K$. Имеет место следуюшая 
Tеорема $2^{\prime}$. Для совершенной коалгебры $K$ пространство $J H_{*}(F K)$ неразложимых әлементов в гомологиях $H_{*}(F K)$ изоморфно пространству примитивных әлементов $P K$ коалгебры $K$.

Действительно, по определению неразложимых элементов $A_{\infty}$-алгебры имеем

$$
J H_{*}(F K)=\operatorname{Im}\left\{H_{*}(F K) \rightarrow H_{*}\left(B H_{*}(F K)\right)\right\} .
$$

Используя изоморфизм $H_{*}\left(B H_{*}(F K)\right) \cong K$, получаем требуемый изоморфизм

$$
J H_{*}(F K) \cong \operatorname{Im}\left\{H_{*}(F K) \rightarrow K\right\} \cong P K .
$$

Заметим, что если гомологии $A_{*}=H_{*}(B A)$ алгебры $A$ являются градуированным пространством со свойством аппроксимации, то когомологии $A^{*}=H^{*}(B A)$ будут $A_{\infty}$-алгеброй, причем пространство неразложимых элементов $J A^{*}$ этой $A_{\infty}$-алгебры будет изоморфно пространству $\overline{P A}_{*}$, двойственному к пространству примитивных элементов $P A_{*}$ в $A_{\infty}$-коалгебре $A_{*}$. Если при этом $A$ - совершенная алгебра, то пространство неразложимых элементов в когомологиях алгебры $A$ изоморфно пространству одномерных когомологий, $J H^{*}(B A) \cong H^{1}(B A)$.

Рассмотрим теперь совершенную коалгебру $K$ и гомологии ее ко- $B$-конструкции $A_{*}=H_{*}(F K)$. Как показано в теореме $1^{\prime}$, пространство неразложимых элементов $A_{*}$ изоморфно пространству $P K=H_{1}(F K)$ примитивных элементов коалгебры $K$. Таким образом, для описания гомологий ко- $B$-конструкции осталось найти соотношения между операциями в $H_{*}(F K)$.

Обозначим через $\nabla(n): K \rightarrow K^{\otimes n}$ итерацию коумножения $\nabla: K \rightarrow K \otimes K$ и через $p: K \rightarrow P K$ проекцию на пространство примитивных элементов. Имеет место следующая

ТеОРема 3. Для совершенной коалгебры $K$ соотношения в $A_{\infty}$-алгебре $A_{*}=H_{*}(F K)$ записываются в виде

$$
\sum_{n \geqslant 0} \pi_{n}(p \otimes \cdots \otimes p) \nabla(n+2)(x)=0, \quad x \in K .
$$

Для доказательства требуемой формулы нужно вычислить образ отображения $K \cong H_{*}\left(B A_{*}\right) \rightarrow A_{\infty}^{1} * A_{*}$. Соответствующие вычисления проведены в работе [3].

Заметим, что для произвольных элементов $x_{1}, \ldots, x_{n} \in P K=H_{1}(F K)$ элементы $\pi_{n-2}\left(x_{1} \otimes \cdots \otimes x_{n}\right)$ принадлежат $H_{2}(F K)$. Поэтому все соотношения, описанные в теореме 2 , являются соотношениями в $H_{2}(F K)$, а соотношения в гомологиях высших размерностей определяются структурой $A_{\infty}$-алгебры в $H_{*}(F K)$. Поэтому имеем

СлЕДСТВИЕ. Для совершенной коалгебры $K$ гомологии $H_{*}(F K)$ определяются гомологиями $H_{1}(F K), H_{2}(F K)$ и операциями $\pi_{n}: H_{1}(F K)^{\otimes n+2} \rightarrow$ $H_{2}(F K)$.

Двойственным образом, для совершенной алгебры $A$ рассмотрим гомологии $K_{*}=H_{*}(B A)$. Как показано в теореме 1 , пространство примитивных элементов $K_{*}$ изоморфно пространству $J A=H_{1}(B A)$ неразложимых элементов алгебры $A$. Таким образом, для описания гомологий $B$-конструкции осталось найти порождаюшие элементы в $\bar{A}_{\infty}^{1} J A$. Обозначим через $q: J A \rightarrow A$ отображение вложения неразложимых элементов алгебры $A$ в саму алгебру $A$ и через $\pi(n): A^{\otimes n} \rightarrow A$ итерацию умножения $\pi: A \otimes A \rightarrow A$. 
Теорема $3^{\prime}$. Для совершенной алгебры $A$ порождающие әлементы в $A_{\infty}$-коалгебре $H_{*}(B A)$ записьвваются в виде

$$
\sum_{n \geqslant 0} \nabla_{n}\left(x_{1} \otimes \cdots \otimes x_{n+2}\right)
$$

где әлементы $x_{1}, \ldots, x_{n+2} \in J A$ таковы, что $\sum_{n \geqslant 0} \pi(n+2) \times$ $\left(x_{1} \otimes \cdots \otimes x_{n+2}\right)=0$.

СлеДСТВИЕ. Для совершенной алгебры $A$ гомологии $H_{*}(B A)$ определяются гомологиями $H_{1}(B A), H_{2}(B A)$ и операциями $\nabla_{n}: H_{2}(B A) \rightarrow H_{1}(B A)^{\otimes n+2}$.

Теорема $3^{\prime \prime}$. Если $A$ - совершенная алгебра и гомологии $A_{*}=H_{*}(B A)$ являются градуированным пространством со свойством аппроксимации, то соотношения в когомологиях $A^{*}=H^{*}(B A)$, рассматриваемых как $A_{\infty}$-алгебра, записьваются в виде

$$
\sum_{n \geqslant 0} \pi_{n}(\overline{q \otimes \cdots \otimes q}) \bar{\pi}(n+2)(x)=0, \quad x \in \bar{A}
$$

Отметим, что вместо условия совершенности алгебр и коалгебр можно накладывать условие гомологической совершенности. Алгебра $A$ и коалгебра $K$ называются гомологически совершенными, если соответствуюшие отображения $A \rightarrow \hat{A}, \breve{K} \rightarrow K$ индуцируют изоморфизм гомологий. Ясно, что приведенные выше теоремы относительно образующих элементов и соотношений будут справедливы и в этом случае.

В качестве примеров описания когомологий алгебр рассмотрим следующие.

1. $A=l_{1}^{m}$. Ясно, что эта алгебра является совершенной. В $H^{*}(A)$ имеется одна образуюшая $u_{1} \in H^{1}(A)$, соответствуюшая элементу $e_{1} \in l_{1}^{m}$, и соотношения

$$
\pi_{n-2}\left(u_{1} \otimes \cdots \otimes u_{1}\right)=0, \quad 2 \leqslant n \leqslant m .
$$

Из этого описания следует, что одномерные когомологии $H^{1}(A)$ изоморфны $\mathbb{C}$. Двумерные когомологии изоморфны $\mathbb{C}$ и имеют одну образующую $u_{2}=$ $\pi_{m-1}\left(u_{1} \otimes u_{1}\right)$. Четномерные когомологии изоморфны $\mathbb{C}$ и имеют образуюшие $u_{2}^{n}=u_{2} \ldots u_{2} \in H^{2 n}(A)$. Нечетномерные когомологии изоморфны $\mathbb{C}$ и имеют образуюшие $u_{2}^{n} \cdot u_{1} \in H^{2 n+1}(A)$.

2. $A=l_{1}$. Эта алгебра не является совершенной. Соответствующая алгебра $\hat{l}_{1}$ состоит из всех формальных рядов с комплексньми коэффициентами. В когомологиях $H^{*}\left(\hat{l}_{1}\right)$ имеется одна образующая $u_{1} \in H^{1}\left(\hat{l}_{1}\right)$, соответствующая элементу $e_{1} \in l_{1}$, и соотношения

$$
\pi_{n-2}\left(u_{1} \otimes \cdots \otimes u_{1}\right)=0, \quad n \geqslant 2 .
$$

Из этого описания следует, что одномерные когомологии $H^{1}\left(\hat{l}_{1}\right)$ изоморфны $\mathbb{C}$ и все высшие когомологии равны нулю. С другой стороны, используя изоморфизм $H^{*}(A) \cong \operatorname{Ext}_{A}(\mathbb{C}, \mathbb{C})$, легко видеть, что $H^{1}\left(l_{1}\right) \cong \mathbb{C}$ и $H^{n}\left(l_{1}\right)=0, n \geqslant 2$. Таким 
образом, отображение $l_{1} \rightarrow \hat{l}_{1}$ индуцирует изоморфизм когомологий, и, следовательно, $l_{1}$ - гомологически совершенная алгебра.

3. $A=l_{1}^{(m)}$. Из точности последовательности $0 \rightarrow l_{1}^{(m)} \rightarrow l_{1} \rightarrow l_{1}^{m} \rightarrow 0$ и гомологической совершенности алгебр $l_{1}$ и $l_{1}^{m}$ следует гомологическая совершенность алгебры $l_{1}^{(m)}$.

В когомологиях $H^{1}(A)$ имеются образуюшие $u_{m+1}, \ldots, u_{2 m+1}$, соответствующие элементам $e_{m+1}, \ldots, e_{2 m+1} \in A$. Соотношения имеют вид

$$
\begin{aligned}
& u_{m+1} u_{m+1}=0, \\
& u_{m+1} u_{m+2}+u_{m+2} u_{m+1}=0 \text {, }
\end{aligned}
$$

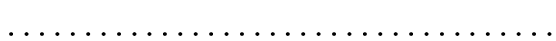

$$
\begin{aligned}
& u_{m+1} u_{2 m+1}+\cdots+u_{2 m+1} u_{m+1}=0 \\
& \pi_{1}\left(u_{m+1} \otimes u_{m+1} \otimes u_{m+1}\right)+u_{m+2} u_{2 m+1}+\cdots+u_{2 m+1} u_{m+2}=0 \text {, }
\end{aligned}
$$

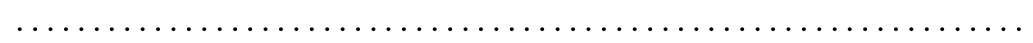

4. $A=\mathscr{O}_{z_{0}}(\mathscr{D})$. Аналогично предыдущему показывается, что эта алгебра является гомологически совершенной. В когомологиях $H^{*}(A)$ имеется одна образующая $u \in H^{1}(A)$, соответствуюшая элементу $z-z_{0} \in A$, и соотношения

$$
\pi_{n-2}(u \otimes \cdots \otimes u)=0, \quad n \geqslant 2
$$

Из этого описания следует, что одномерные когомологии $H^{1}(A)$ изоморфны $\mathbb{C}$ и все высшие когомологии равны нулю.

\section{Список литературы}

1. Gugenheim V. K., May J. P. On the theory and applications of differential torsion products // Mem. AMS. 1974. V. 142. P. 1-93.

2. Stasheff J. D. Homotopy associativity of $H$-spaces // Trans. Amer. Math. Soc. 1963. V. 108. № 2. P. 275-312.

3. Смирнов $B$. $A$. Гомологии $B$-конструкций и ко- $B$-конструкций // Изв. РАН. Сер. матем. 1994. T. 58. № 4. C. $80-96$.

4. Хелемский A. Я. Банаховы и полинормированные алгебры. М.: Наука, 1989.

Поступило в редакцию 17.X.1996 